\title{
Microbiological Quality of Peanuts: From Field to Consumption
}

$$
\text { Okşan UÇKUN }{ }^{1, a^{*}} \text {, Işıl VAR }{ }^{2, b}
$$

\author{
${ }^{1}$ Adana Food Control Laboratory Directorate, Republic of Turkey Ministry of Food, Agriculture and \\ Livestock, Adana, Turkey \\ ${ }^{2}$ Faculty of Agriculture, Department of Food Engineering, Cukurova University, Adana, Turkey \\ auckun_oksan@hotmail.com, bivar@cu.edu.tr
}

Keywords: Peanut (Arachis hypogaea L.), food safety, microbiology, pathogens

\begin{abstract}
Peanuts, including shelled peanuts, unshelled peanuts and roasted peanuts which are ready to eat foods, recently appeared as possible sources of infection with foodborne bacteria besides the high levels of aflatoxins. Peanuts which are taken from harvest, storage and snack shops were evaluated for the presence of Listeria spp. and Salmonella spp; also total aerobic mesophilic bacteria, coliforms, E.coli. and yeasts and moulds, were enumerated. A total of 92 samples were found to be clean in terms of Salmonella and E-coli O157: H7, but only in 1 of the samples of shelled peanut taken from harvest found Listeria spp. Shelled peanut samples collected from harvest and storage were found to be contaminated in terms of microorganisms, however, microbiological contamination was found to be less in the samples obtained when the shells of the same specimens were removed. In addition, in terms of microorganisms it was observed that the shell free samples taken from storage were dirtier than the samples which was being removed the shell taken from storage. Shell-free (unpackaged) roasted peanuts samples purchased from the snack shops was found clean in terms of the pathogens. When salt and heat treatment applied over $4 \%$, existing microorganisms can be damaged. Besides, the water activity value of the product can reduce the level of that microorganisms growth. Therefore, processed peanuts are safer in the environment in which they are stored, unless the relative humidity increase and is not subject to crosscontamination.
\end{abstract}

\section{Introduction}

Peanuts (Arachis hypogaea L.) are one of the most important oilseed crops and snack foods in the world Agro-food trade market. The major producers/exporters of peanuts are the United States, China, Argentina, Sudan, Senegal, and Brazil. Peanuts are globally used as an economical food source as they contain high quality proteins, unsaturated fatty acid, and are rich in minerals. Peanuts are consumed whole roasted and boiled, and as ingredients in a variety forms of products include peanut brittle, peanut butter, and peanut oil, and so on [1].

In Turkey, the areas where peanut planting and production areas are most common are Adana and Osmaniye $(80 \%)$. The plants are first excavated from the soil by special plows and the plants are drawn by the workers coming from behind and the fruits are laid on top of the soil. After the plants are kept in this way for up to three days in the field (when the ratio of peanuts to moisture falls to 20\%), fruits are separated from their stalks by harvesting machines. Peanuts are often contaminated with different microorganisms from different environmental sources, such as soil, cattle or other animals and insects, reptiles, human resources when hygiene procedures are not performed at all or performed at little, in the post-harvest processes of conventional harvest and peanuts.

Peanuts can be consumed both raw or roasted in our country. Seeds are roasted after the outer shell is not broken or the shell is broken and it is presented for consumption. Peanuts can be sold as packaged and unpackaged in snack shops and grocery stores in room temperature. And it is sold also raw shelled peanuts on the street. These conditions make the peanut vulnerable to the microbiological risks. So far although there have not been studies on the microbiological characteristics of the peanut, which is thought to be clean from a microbiological point of view, since 1960, studies only on aflatoxin, a mycotoxin that the moulds have produced, have been 
extensively carried out. However, almonds, cashews, coconut, hazelnuts, pine nuts, pecans, pistachios, sesame seeds, and peanuts, as well as several processed nut products, have been associated with foodborne outbreaks and/or recalls after isolation of foodborne pathogens. It was seen that, the majority of these outbreaks and recalls have been related with Salmonella, and many of the outbreaks have lasted for months and have included cases from multiple states in the United States and/or from other countries. Therefore, most published surveys have focused on indicator organisms and Salmonella. In the United Kingdom, the incidence of Salmonella on retail edible nuts and seeds was under 1\%; samples included roasted nut kernels (25 g, n 727, 11 nut species, processed (heat or added preservatives) nuts (25 g, $\mathrm{n} \sim 2,886,12$ nut species) and dried seeds ( $25 \mathrm{~g}, \mathrm{n}$ $\sim 3,735)$. The prevalence of Salmonella on raw almond kernels was about $1 \%$ over 8 years $(100 \mathrm{~g}, \mathrm{n}$ $\sim$ 13,972) [2]. Outbreaks and recalls due to Escherichia coli O157:H7 and recalls due to Listeria monocytogenes contamination also are documented for some nuts and nut products [3].

Low $\mathrm{a}_{\mathrm{w}}$ foods have usually been considered safe regarding foodborne pathogens because the optimum $\mathrm{a}_{\mathrm{w}}$ for growth of these pathogens is over 0.95 . But, unfortunately, there have been several outbreaks were seen also due to foods which were low water activity contaminated with $E$. coli O157:H7, S. Typhimurium, and L. monocytogenes [4]. Dry foods like peanuts do not often support microbial growth, but they may still be able to allow survival of pathogens. Komitopoulou and Penaloza (2009) found that inoculated Salmonella could survive for 3-4 weeks on dry, raw materials including crushed cocoa and hazelnut shells, cocoa beans, and almond kernels at both room temperature and $5^{\circ} \mathrm{C}$. Salmonella is typically inhibited by $\mathrm{a}_{\mathrm{w}}<0.91$, but the aw of these substances were likely much lower [5].

Pathogens can be transferred in several ways to food, such as through contaminated water and equipment, poor worker hygiene, and pests [5]. At harvest, if good agricultural and hygiene practices are followed, nuts are harvested with minimal damage and then dried to a water activity of less than 0.7. The Codex International Code of Hygienic Practice for tree nuts states that these products should be free from pathogenic microorganisms [6]. Although nut-associated outbreaks of infection are relatively uncommon, recent outbreaks of salmonellosis associated with the consumption of peanuts, peanut products, and almonds have raised awareness of nuts as a potential vehicle for foodborne illness [7]. For this reason, this study was designed to evaluate the microbiological quality of the peanuts produced in our country. This study was the first investigation the microbiological quality of peanuts in our country and peanut was evaluated for pathogens such as Salmonella, Listeria spp., Coliform and E. coli, as well as mold, yeast and total aerobic mesophilic bacteria.

\section{Materials and Methods}

Peanut samples supplied from the producers in Osmaniye during harvesting period and storage. Also, roasted peanuts were purchased from snack shops in Adana and Osmaniye. In the harvesting period, a total of 24 samples (12 shelled samples and 12 removed the shell samples) collected from different fields, a total of 36 samples (12 shelled samples and 12 removed the shell samples and 12 shell-free samples) taken from storage and 32 shell-free (unpackaged) roasted samples from snack shops, a total of 92 samples has been studied.

Total aerobic mesophilic bacteria were enumerated at Plate Count Agar (Merck). PCA plates were incubated for $48 \mathrm{~h}$ at $30^{\circ} \mathrm{C}$. Potato Dextrose Agar (Merck) was used for isolate yeast and mould and incubated at $25^{\circ} \mathrm{C}$ for seven days [8].

Enumeration of coliform and E.coli were performed with Most Probable Number (MPN) technique and used Fluorocult Lauryl Sulfate Broth (Merck). Fluorocult Laurly Sulphate Broth tubes were incubated at $37{ }^{\circ} \mathrm{C}$ for $24-48 \mathrm{~h}$ incubation. After examining tubes of gas forming with UV light and applied Kovac's indole separator for indole test. Fluorescent positive and indole positive tubes as Escherichia coli type 1, fluorescent negative and indol positive tubes as E. coli O157: H7 were determined [9-10]. E. coli O157: H7 confirmation was also done using Fluorocult E. coli $\mathrm{O} 157: \mathrm{H7}$ Agar. 
Analyses of Salmonella spp. were carried out in four steps. Briefly, 25 grams of samples were weighed in $225 \mathrm{ml}$ of Buffered Peptone Water (Merck) for non-selective pre enrichment step for $16-20 \mathrm{~h}$ at $37^{\circ} \mathrm{C}$. Following the incubation, for selective enrichment step $0.1 \mathrm{~mL}$ and $10 \mathrm{~mL}$ were transferred to $10 \mathrm{~mL}$ of Rappaport Vassiliadis Broth (Merck) and $100 \mathrm{~mL}$ of Selenite Cystein Broth (Merck) and incubated at $42^{\circ} \mathrm{C}$ and $37^{\circ} \mathrm{C}$ for $24 \mathrm{~h}$, respectively. After incubation a loopful were streaked on both Xylose Lysine Deoxycholate (XLD) Agar and Hektoen Enteric Agar (Merck), and incubated at $37^{\circ} \mathrm{C}$ for $24-48 \mathrm{~h}$. Colonies isolated from each media were further analysed by biochemical reactions (lactose, gas from glucose, $\mathrm{H}_{2} \mathrm{~S}$, urea, indole, citrate, lysine decarboxylase).

Listeria spp. isolation was performed according to FDA. The analyzed sample was weighed at 25 grams and incubated for $4 \mathrm{~h}$ at $30{ }^{\circ} \mathrm{C}$ in $225 \mathrm{ml}$ Buffered Listeria Enrichment Broth (BLEB) medium for pre-enrichment. After $4 \mathrm{~h}$, selective addition $(10 \mathrm{mg} / 1$ acriflavine, $40 \mathrm{mg} / 1$ nalidixic acid) the incubation was completed in 48 hours. At the 24th and 48th hours from the beginning of the incubation, the samples was taken from BLEB medium to PALCAM Agar and the petri dishes were left incubation at $37^{\circ} \mathrm{C}$ for 48 hours. After incubation, on PALCAM agar gray-green or black colonies was suspected as Listeria and was taken and spiked to Tryptic Soy Agar-Yeast medium to purify. After incubation, colony and cell morphology of these cells were evaluated. Gram positive, catalase positive, oxidase negative, mobility positive and urea negative colonies were determined as Listeria spp. positive [11-12].

\section{Results and Discussion}

A total of 92 samples taken from harvest, storage and snack shops were found to be clean in terms of Salmonella and E-coli O157: H7, but only in 1 of the samples of shelled peanut taken from harvest found Listeria. The analysis results are given in Tables 1-6 below.

Table 1. Microbiological analysis results of shelled peanut samples taken from harvested.

\begin{tabular}{|c|c|c|c|c|c|c|c|c|}
\hline Samples & $\begin{array}{l}\text { Coliform } \\
\text { (MPN/g) }\end{array}$ & $\begin{array}{l}\text { E.coli } \\
\text { Tip 1 }\end{array}$ & $\begin{array}{l}\text { E-coli } \\
\text { O157:H7 }\end{array}$ & Salmonella & $\begin{array}{l}\text { Listeria } \\
\text { spp. }\end{array}$ & $\begin{array}{c}\text { Yeast } \\
\text { (log } \\
\text { kob/g) }\end{array}$ & $\begin{array}{l}\text { Motal } \\
\text { Mould } \\
\text { (log kob/g) }\end{array}$ & $\begin{array}{l}\text { aerobic } \\
\text { mesophilic } \\
\text { bacteria } \\
\text { kob/g) }\end{array}$ \\
(log \\
\hline 1 & 240 & 240 & $<3$ & N.D. & N.D. & $4.7 \pm 0.1$ & N.D. & $3.0 \pm 0.1$ \\
\hline 2 & 460 & 460 & $<3$ & N.D. & N.D. & $3.9 \pm 0.2$ & $3.0 \pm 0.2$ & $4.5 \pm 0.1$ \\
\hline 3 & 9,1 & 9,1 & $<3$ & N.D. & N.D. & $5.1 \pm 0.1$ & N.D. & $5.0 \pm 0.2$ \\
\hline 4 & 15 & $<3$ & $<3$ & N.D. & N.D. & $3.7 \pm 0.3$ & $3.6 \pm 0.1$ & $4.3 \pm 0.3$ \\
\hline 5 & 23 & $<3$ & $<3$ & N.D. & N.D. & $4.0 \pm 0.2$ & $2.7 \pm 0.3$ & $4.3 \pm 0.2$ \\
\hline 6 & 460 & $<3$ & $<3$ & N.D. & N.D. & $3.6 \pm 0.3$ & N.D. & $4.3 \pm 0.1$ \\
\hline 7 & 23 & $<3$ & $<3$ & N.D. & N.D. & $5.3 \pm 0.1$ & $3.4 \pm 0.1$ & $5.0 \pm 0.1$ \\
\hline 8 & $>1100$ & 240 & $<3$ & N.D. & N.D. & $4.7 \pm 0.3$ & $5.3 \pm 0.1$ & $5.6 \pm 0.2$ \\
\hline 9 & 290 & $<3$ & $<3$ & N.D. & N.D. & $3.6 \pm 0.2$ & $5.6 \pm 0.2$ & $6.9 \pm 0.3$ \\
\hline 10 & $>1100$ & $>1100$ & $<3$ & N.D. & Positive & $8.7 \pm 0.2$ & N.D. & $8.7 \pm 0.1$ \\
\hline 11 & 39 & $<3$ & $<3$ & N.D. & N.D. & $3.8 \pm 0.1$ & $4.6 \pm 0.3$ & $5.5 \pm 0.1$ \\
\hline 12 & 20 & $<3$ & $<3$ & N.D. & N.D. & $3.7 \pm 0.1$ & $3.4 \pm 0.1$ & $4.4 \pm 0.3$ \\
\hline
\end{tabular}


Table 2. Microbiological analysis results taken from harvested period samples that were being removed the shell.

\begin{tabular}{|c|c|c|c|c|c|c|c|c|}
\hline Samples & $\begin{array}{l}\text { Coliform } \\
\text { (MPN/g) }\end{array}$ & $\begin{array}{l}\text { E.coli } \\
\text { Tip 1 }\end{array}$ & $\begin{array}{l}\text { E-coli } \\
\text { O157: } \\
\text { H7 }\end{array}$ & Salmonella & $\begin{array}{l}\text { Listeria } \\
\text { spp. }\end{array}$ & $\begin{array}{l}\text { Yeast } \\
\text { (log } \\
\mathbf{k o b} / \mathbf{g})\end{array}$ & $\begin{array}{l}\text { Mould } \\
\text { (log } \\
\mathbf{k o b} / \mathbf{g})\end{array}$ & $\begin{array}{l}\text { Total aerobic } \\
\text { mesophilic } \\
\text { bacteria } \\
\text { kob/g) }\end{array}$ \\
\hline 1 & $<3$ & $<3$ & $<3$ & N.D. & N.D. & $2.0 \pm 0.1$ & $3.0 \pm 0.3$ & $3.4 \pm 0.1$ \\
\hline 2 & $<3$ & $<3$ & $<3$ & N.D. & N.D. & $2.7 \pm 0.1$ & N.D. & $3.6 \pm 0.2$ \\
\hline 3 & $<3$ & $<3$ & $<3$ & N.D. & N.D. & $3.6 \pm 0.3$ & $3.0 \pm 0.2$ & $4.5 \pm 0.3$ \\
\hline 4 & $<3$ & $<3$ & $<3$ & N.D. & N.D. & N.D. & $3.9 \pm 0.1$ & $4.3 \pm 0.1$ \\
\hline 5 & $<3$ & $<3$ & $<3$ & N.D. & N.D. & $2.5 \pm 0.1$ & N.D. & $3.8 \pm 0.1$ \\
\hline 6 & $<3$ & $<3$ & $<3$ & N.D. & N.D. & $2.6 \pm 0.2$ & $3.3 \pm 0.1$ & $3.5 \pm 0.3$ \\
\hline 7 & $<3$ & $<3$ & $<3$ & N.D. & N.D. & $3.6 \pm 0.1$ & $2.7 \pm 0.2$ & $3.8 \pm 0.2$ \\
\hline 8 & $<3$ & $<3$ & $<3$ & N.D. & N.D. & $2.9 \pm 0.2$ & $2.7 \pm 0.1$ & $4.0 \pm 0.1$ \\
\hline 9 & $<3$ & $<3$ & $<3$ & N.D. & N.D. & $5.2 \pm 0.1$ & N.D. & $5.6 \pm 0.3$ \\
\hline 10 & $<3$ & $<3$ & $<3$ & N.D. & N.D. & $2.4 \pm 0.1$ & $3.4 \pm 0.2$ & $3.7 \pm 0.2$ \\
\hline 11 & $<3$ & $<3$ & $<3$ & N.D. & N.D. & $3.3 \pm 0.2$ & $2.6 \pm 0.3$ & $5.2 \pm 0.1$ \\
\hline 12 & $<3$ & $<3$ & $<3$ & N.D. & N.D. & $2.7 \pm 0.3$ & $3.0 \pm 0.1$ & $3.4 \pm 0.1$ \\
\hline
\end{tabular}

Table 3. Microbiological analysis results of shelled peanut samples taken from storage.

\begin{tabular}{|c|c|c|c|c|c|c|c|c|}
\hline Samples & $\begin{array}{l}\text { Coliform } \\
\text { (MPN/g) }\end{array}$ & $\begin{array}{l}\text { E.coli } \\
\text { Tip 1 }\end{array}$ & $\begin{array}{l}\boldsymbol{E}- \\
\text { coli } \\
\mathbf{O 1 5 7} \\
\mathbf{: H 7}\end{array}$ & Salmonella & $\begin{array}{l}\text { Listeria } \\
\text { spp. }\end{array}$ & $\begin{array}{l}\text { Yeast } \\
\text { (log } \\
\mathbf{k o b} / \mathbf{g})\end{array}$ & $\begin{array}{l}\text { Mould } \\
\text { (log } \\
\mathbf{k o b} / \mathbf{g})\end{array}$ & $\begin{array}{l}\text { Total aerobic } \\
\text { mesophilic } \\
\text { bacteria } \\
\text { kob/g) }\end{array}$ \\
\hline 1 & $>1100$ & $>1100$ & $<3$ & N.D. & N.D. & $7.3 \pm 0.1$ & $3.3 \pm 0.2$ & $7.4 \pm 0.1$ \\
\hline 2 & $<3$ & $<3$ & $<3$ & N.D. & N.D. & $3.9 \pm 0.2$ & $3.4 \pm 0.1$ & $4.8 \pm 0.2$ \\
\hline 3 & 23 & 9,1 & $<3$ & N.D. & N.D. & $5.7 \pm 0.1$ & $5.0 \pm 0.3$ & $6.1 \pm 0.3$ \\
\hline 4 & 23 & $<3$ & $<3$ & N.D. & N.D. & N.D. & N.D. & $3.1 \pm 0.2$ \\
\hline 5 & $>1100$ & 35 & $<3$ & N.D. & N.D. & N.D. & N.D. & N.D. \\
\hline 6 & 39 & 3,6 & $<3$ & N.D. & N.D. & N.D. & N.D. & $3.3 \pm 0.1$ \\
\hline 7 & $>1100$ & $>1100$ & $<3$ & N.D. & N.D. & $4.7 \pm 0.1$ & $3.4 \pm 0.2$ & $4.9 \pm 0.2$ \\
\hline 8 & $>1100$ & 15 & $<3$ & N.D. & N.D. & $5.2 \pm 0.1$ & $4.6 \pm 0.1$ & $5.5 \pm 0.1$ \\
\hline 9 & 7,2 & $<3$ & $<3$ & N.D. & N.D. & N.D. & N.D. & N.D. \\
\hline 10 & $<3$ & $<3$ & $<3$ & N.D. & N.D. & $3.6 \pm 0.1$ & $3.0 \pm 0.2$ & $4.6 \pm 0.3$ \\
\hline 11 & $<3$ & $<3$ & $<3$ & N.D. & N.D. & $3.4 \pm 0.2$ & $3.0 \pm 0.1$ & $3.8 \pm 0.1$ \\
\hline 12 & 15 & 6 & $<3$ & N.D. & N.D. & $4.7 \pm 0.1$ & $3.3 \pm 0.2$ & $5.0 \pm 0.2$ \\
\hline
\end{tabular}


Table 4. Microbiological analysis results of samples that were being removed the shell taken from storage.

\begin{tabular}{|c|c|c|c|c|c|c|c|c|}
\hline Samples & $\begin{array}{l}\text { Coliform } \\
\text { (MPN/g) }\end{array}$ & $\begin{array}{l}\text { E.coli } \\
\text { Tip 1 }\end{array}$ & $\begin{array}{l}\boldsymbol{E}- \\
\text { coli } \\
\mathbf{O 1 5 7} \\
\mathbf{: H 7}\end{array}$ & Salmonella & $\begin{array}{c}\text { Listeria } \\
\mathbf{s p p .}\end{array}$ & $\begin{array}{c}\text { Yeast } \\
\text { (log } \\
\mathbf{k o b} / \mathbf{g})\end{array}$ & $\begin{array}{l}\text { Mould } \\
\mathbf{l o g} \\
\mathbf{k o b} / \mathbf{g})\end{array}$ & $\begin{array}{l}\text { Total aerobic } \\
\text { mesophilic } \\
\text { bacteria } \\
\text { kob/g) }\end{array}$ \\
\hline 1 & $<3$ & $<3$ & $<3$ & N.D. & N.D. & $3.1 \pm 0.2$ & $3.7 \pm 0.1$ & $4.1 \pm 0.3$ \\
\hline 2 & $<3$ & $<3$ & $<3$ & N.D. & N.D. & N.D. & N.D. & $4.9 \pm 0.2$ \\
\hline 3 & $<3$ & $<3$ & $<3$ & N.D. & N.D. & $3.5 \pm 0.2$ & $3.2 \pm 0.2$ & $3.7 \pm 0.2$ \\
\hline 4 & $<3$ & $<3$ & $<3$ & N.D. & N.D. & $2.7 \pm 0.3$ & $2.3 \pm 0.3$ & $3.0 \pm 0.1$ \\
\hline 5 & $<3$ & $<3$ & $<3$ & N.D. & N.D. & $2.6 \pm 0.1$ & $1.6 \pm 0.1$ & $4.2 \pm 0.3$ \\
\hline 6 & $<3$ & $<3$ & $<3$ & N.D. & N.D. & $3.2 \pm 0.1$ & N.D. & $4.4 \pm 0.2$ \\
\hline 7 & $<3$ & $<3$ & $<3$ & N.D. & N.D. & $3.6 \pm 0.2$ & $2.0 \pm 0.3$ & $3.7 \pm 0.1$ \\
\hline 8 & 150 & $<3$ & $<3$ & N.D. & N.D. & $4.8 \pm 0.2$ & $3.6 \pm 0.2$ & $3.5 \pm 0.1$ \\
\hline 9 & $<3$ & $<3$ & $<3$ & N.D. & N.D. & $3.6 \pm 0.3$ & N.D. & $3.7 \pm 0.3$ \\
\hline 10 & $<3$ & $<3$ & $<3$ & N.D. & N.D. & N.D. & N.D. & $3.0 \pm 0.2$ \\
\hline 11 & $<3$ & $<3$ & $<3$ & N.D. & N.D. & N.D. & N.D. & $2.0 \pm 0.2$ \\
\hline 12 & $<3$ & $<3$ & $<3$ & N.D. & N.D. & $2.3 \pm 0.1$ & N.D. & $2.8 \pm 0.3$ \\
\hline
\end{tabular}

Table 5. Microbiological analysis results of shell-free peanuts samples taken from storage.

\begin{tabular}{|c|c|c|c|c|c|c|c|c|}
\hline Samples & $\begin{array}{l}\text { Coliform } \\
\text { (MPN/g) }\end{array}$ & $\begin{array}{l}\text { E.coli } \\
\text { Tip 1 }\end{array}$ & $\begin{array}{l}\text { E-coli } \\
\text { O157: } \\
\text { H7 }\end{array}$ & Salmonella & $\begin{array}{c}\text { Listeria } \\
\text { spp. }\end{array}$ & $\begin{array}{c}\text { Yeast } \\
\text { (log } \\
\mathbf{k o b} / \mathbf{g})\end{array}$ & $\begin{array}{l}\text { Total aerobic } \\
\text { mesophilic } \\
\text { (log kob/g) } \\
\text { bacteria } \\
\text { kob/g) }\end{array}$ \\
\hline 1 & 93 & $<3$ & $<3$ & N.D. & N.D. & N.D. & N.D. & N.D. \\
\hline 2 & 9,1 & 9,1 & $<3$ & N.D. & N.D. & N.D. & N.D. & N.D. \\
\hline 3 & $<3$ & $<3$ & $<3$ & N.D. & N.D. & N.D. & N.D. & N.D. \\
\hline 4 & 1100 & $<3$ & $<3$ & N.D. & N.D. & $5.3 \pm 0.2$ & $3.1 \pm 0.2$ & $5.7 \pm 0.2$ \\
\hline 5 & 21 & 21 & $<3$ & N.D. & N.D. & $4.7 \pm 0.1$ & $2.1 \pm 0.3$ & $4.8 \pm 0.1$ \\
\hline 6 & $<3$ & $<3$ & $<3$ & N.D. & N.D. & $4.4 \pm 0.1$ & N.D. & $5.1 \pm 0.3$ \\
\hline 7 & $>1100$ & 36 & $<3$ & N.D. & N.D. & $4.7 \pm 0.2$ & N.D. & $5.3 \pm 0.2$ \\
\hline 8 & 15 & 3 & $<3$ & N.D. & N.D. & $3.4 \pm 0.3$ & $3.1 \pm 0.1$ & $2.9 \pm 0.1$ \\
\hline 9 & $<3$ & $<3$ & $<3$ & N.D. & N.D. & $4.9 \pm 0.2$ & N.D. & $5.3 \pm 0.1$ \\
\hline 10 & 36 & $<3$ & $<3$ & N.D. & N.D. & N.D. & N.D. & N.D. \\
\hline 11 & $>1100$ & 3 & $<3$ & N.D. & N.D. & $5.0 \pm 0.3$ & N.D. & $5.2 \pm 0.1$ \\
\hline 12 & $<3$ & $<3$ & $<3$ & N.D. & N.D. & $5.2 \pm 0.2$ & N.D. & $5.5 \pm 0.2$ \\
\hline
\end{tabular}


Table 6. Microbiological analysis results of shell-free roasted peanuts samples taken from snack shops.

\begin{tabular}{|c|c|c|c|c|c|c|c|c|}
\hline Samples & $\begin{array}{l}\text { Coliform } \\
\text { (MPN/g) }\end{array}$ & $\begin{array}{l}\text { E.coli } \\
\text { Tip } 1\end{array}$ & $\begin{array}{l}\text { E-coli } \\
\text { O157:H7 }\end{array}$ & Salmonella & $\begin{array}{l}\text { Listeria } \\
\text { spp. }\end{array}$ & $\begin{array}{c}\text { Yeast } \\
(\log \\
\text { kob/g) }\end{array}$ & $\begin{array}{l}\text { Mould } \\
(\log \\
\text { kob/g) }\end{array}$ & $\begin{array}{l}\text { Total aerobic } \\
\text { mesophilic } \\
\text { bacteria (log } \\
\text { kob/g) }\end{array}$ \\
\hline 1 & $<3$ & $<3$ & $<3$ & N.D. & N.D. & N.D. & N.D. & $2.0 \pm 0.2$ \\
\hline 2 & $<3$ & $<3$ & $<3$ & N.D. & N.D. & N.D. & N.D. & $3.6 \pm 0.1$ \\
\hline 3 & $<3$ & $<3$ & $<3$ & N.D. & N.D. & N.D. & N.D. & $3.6 \pm 0.3$ \\
\hline 4 & $<3$ & $<3$ & $<3$ & N.D. & N.D. & N.D. & N.D. & $2.6 \pm 0.1$ \\
\hline 5 & $<3$ & $<3$ & $<3$ & N.D. & N.D. & N.D. & N.D. & $2.1 \pm 0.2$ \\
\hline 6 & $<3$ & $<3$ & $<3$ & N.D. & N.D. & N.D. & N.D. & $2.3 \pm 0.2$ \\
\hline 7 & $<3$ & $<3$ & $<3$ & N.D. & N.D. & N.D. & N.D. & $3.6 \pm 0.3$ \\
\hline 8 & $<3$ & $<3$ & $<3$ & N.D. & N.D. & N.D. & N.D. & N.D. \\
\hline 9 & $<3$ & $<3$ & $<3$ & N.D. & N.D. & N.D. & N.D. & N.D. \\
\hline 10 & $<3$ & $<3$ & $<3$ & N.D. & N.D. & N.D. & N.D. & N.D. \\
\hline 11 & $<3$ & $<3$ & $<3$ & N.D. & N.D. & N.D. & N.D. & N.D. \\
\hline 12 & $<3$ & $<3$ & $<3$ & N.D. & N.D. & N.D. & N.D. & N.D. \\
\hline 13 & $<3$ & $<3$ & $<3$ & N.D. & N.D. & $2.7 \pm 0.1$ & N.D. & $4.4 \pm 0.2$ \\
\hline 14 & $<3$ & $<3$ & $<3$ & N.D. & N.D. & N.D. & N.D. & $4.4 \pm 0.1$ \\
\hline 15 & $<3$ & $<3$ & $<3$ & N.D. & N.D. & $2.9 \pm 0.1$ & $2.4 \pm 0.1$ & $3.5 \pm 0.3$ \\
\hline 16 & 23 & $<3$ & $<3$ & N.D. & N.D. & $3.0 \pm 0.1$ & N.D. & $4.1 \pm 0.2$ \\
\hline 17 & $<3$ & $<3$ & $<3$ & N.D. & N.D. & $4.9 \pm 0.2$ & $2.6 \pm 0.2$ & $5.0 \pm 0.1$ \\
\hline 18 & $<3$ & $<3$ & $<3$ & N.D. & N.D. & $3.2 \pm 0.3$ & N.D. & $3.7 \pm 0.2$ \\
\hline 19 & $<3$ & $<3$ & $<3$ & N.D. & N.D. & $3.6 \pm 0.2$ & $1.6 \pm 0.2$ & $3.7 \pm 0.1$ \\
\hline 20 & $<3$ & $<3$ & $<3$ & N.D. & N.D. & $3.5 \pm 0.1$ & N.D. & $3.6 \pm 0.1$ \\
\hline 21 & $<3$ & $<3$ & $<3$ & N.D. & N.D. & $3.3 \pm 0.1$ & N.D. & $4.3 \pm 0.2$ \\
\hline 22 & $<3$ & $<3$ & $<3$ & N.D. & N.D. & $3.6 \pm 0.2$ & N.D. & $4.2 \pm 0.2$ \\
\hline 23 & $<3$ & $<3$ & $<3$ & N.D. & N.D. & $3.3 \pm 0.2$ & N.D. & $3.5 \pm 0.1$ \\
\hline 24 & $<3$ & $<3$ & $<3$ & N.D. & N.D. & $3.8 \pm 0.1$ & N.D. & $4.0 \pm 0.3$ \\
\hline 25 & $<3$ & $<3$ & $<3$ & N.D. & N.D. & $3.5 \pm 0.1$ & N.D. & $4.2 \pm 0.1$ \\
\hline 26 & $<3$ & $<3$ & $<3$ & N.D. & N.D. & $3.9 \pm 0.2$ & N.D. & $4.7 \pm 0.2$ \\
\hline 27 & $<3$ & $<3$ & $<3$ & N.D. & N.D. & $3.7 \pm 0.1$ & N.D. & $3.8 \pm 0.1$ \\
\hline 28 & $<3$ & $<3$ & $<3$ & N.D. & N.D. & $3.9 \pm 0.2$ & N.D. & $4.3 \pm 0.2$ \\
\hline 29 & $<3$ & $<3$ & $<3$ & N.D. & N.D. & $4.5 \pm 0.1$ & N.D. & $4.7 \pm 0.1$ \\
\hline 30 & $<3$ & $<3$ & $<3$ & N.D. & N.D. & $3.7 \pm 0.3$ & $3.3 \pm 0.1$ & $3.9 \pm 0.3$ \\
\hline 31 & 3 & $<3$ & $<3$ & N.D. & N.D. & $3.9 \pm 0.2$ & $2.6 \pm 0.2$ & $4.2 \pm 0.1$ \\
\hline 32 & $<3$ & $<3$ & $<3$ & N.D. & N.D. & $3.8 \pm 0.1$ & N.D. & $4.3 \pm 0.2$ \\
\hline
\end{tabular}

N.D: not determined

Table 1 shows that microbiological analysis results of shelled peanut samples taken from harvested. A total of 12 shelled peanut samples which were taken from harvest, as the range of in 12 samples total aerobic mesophilic bacteria 3.0 $\pm 0.1-8.7 \pm 0.1 \log \mathrm{CFU} / \mathrm{g}$, in $12 \mathrm{samples}$ coliform group bacteria 9.1->1100 MPN/g, in 12 samples E.coli Type $1<3->1100 \mathrm{MPN} / \mathrm{g}$, in 12 samples yeast 3.6 $\pm 0.2-8.7 \pm 0.2 \log \mathrm{CFU} / \mathrm{g}$, in 8 samples mold 2.7 $\pm 0.3-5.6 \pm 0.2 \log \mathrm{CFU} / \mathrm{g}$ were found. In 1 of the samples of shelled peanut taken from harvest found Listeria spp. When removing the shell of 12 of peanut samples from harvested period (Table 2), total aerobic mesophilic bacteria 3.4 $\pm 0.1-5.6 \pm 0.3$ $\log$ CFU/g in 12 samples, in 11 samples yeast 2.0 $\pm 0.1-5.2 \pm 0.1 \log$ CFU/g, and in 9 samples mold $2.6 \pm 0.3-3.9 \pm 0.1 \log \mathrm{CFU} / \mathrm{g}$ in the range of were found. 
Listeria monocytogenes is a microorganism of significant public concern that can cause severe illness and death in susceptible populations. It was estimated in 1999 that L.monocytogenes causes 500 deaths per year in the United States. Pregnant women, neonates, elderly persons, and immunocompromised patients are most susceptible to listeriosis [13]. Escherichia coli $\mathrm{O} 157: \mathrm{H} 7$ is an increasingly common cause of illness, including bloody diarrhea and hemolytic uremic syndrome [4].

In order to keep pathogens at a minimum level in the peanuts, it is necessary to follow up with the requirements of good agriculture and hygiene practices such as harvesting the peanuts without damaging and drying them to the desired moisture level.

Microbiological analysis results of shelled peanut samples taken from storage are shown in Table 3. In 12 shelled peanut samples which were taken from storage, in 10 of them total aerobic mesophilic bacteria 3.1 $\pm 0.2-7.4 \pm 0.1 \log \mathrm{CFU} / \mathrm{g}$, in 12 samples coliform group bacteria $<3->1100 \mathrm{MPN} / \mathrm{g}$, E.coli Type $1<3->1100 \mathrm{MPN} / \mathrm{g}$, in 8 samples yeast $3.4 \pm 0.2-7.3 \pm 0.1 \mathrm{log} \mathrm{CFU} / \mathrm{g}$, mold 3.0 $\pm 0.1-5.0 \pm 0.3 \mathrm{log} \mathrm{CFU} / \mathrm{g}$ in the range of were found. When removing the shell of 12 of peanut samples (Table 4), were found coliform group bacteria $(150 \mathrm{MPN} / \mathrm{g})$ only in one sample, and E.coli was not found in any of the samples. Besides, total aerobic mesophilic bacteria was found in the range of 2.0 $\pm 0.2-4.9 \pm 0.2 \mathrm{log} C F U / g$ in 12 samples. Also, yeast 2.3 $\pm 0.1-4.8 \pm 0.2 \log$ $\mathrm{CFU} / \mathrm{g}$ in 9 samples, and mold 1.6 $\pm 0.1-3.7 \pm 0.1 \mathrm{log}$ CFU/g in 6 samples were found.

Of the 12 shell-free peanut samples taken from storage, total aerobic mesophilic bacteria 2.9 $\pm 0.1-5.7 \pm 0.2 \mathrm{log}$ CFU/g in 8 samples, coliform group bacteria $<3->1100 \mathrm{MPN} / \mathrm{g}$ in 12 samples as was found (Table 5). Moreover, E.coli Tip1 as $<3-36 \mathrm{MPN} / \mathrm{g}$ in 12 samples, yeast as $3.4 \pm 0.3-$ $5.3 \pm 0.2 \log$ CFU/g in 8 samples, and mold $2.1 \pm 0.3-3.1 \pm 0.2 \log$ CFU/g in 3 samples, were found.

Table 6 shows that microbiological analysis results of shell-free roasted peanut samples. 27 of the 32 peanut samples which purchased from the snack shops, the total aerobic mesophilic bacteria were found to be between $2.0 \pm 0.2-5.0 \pm 0.1 \mathrm{log} C F U / g$. While in 2 samples coliform group bacteria were found to be 3-23 MPN/g and E.coli was not found. In 19 samples, yeast were found to be between 2.7 $\pm 0.1-4.9 \pm 0.2 \mathrm{log} \mathrm{CFU} / \mathrm{g}$ and mold were found to be between 1.6 $\pm 0.2-3.3 \pm 0.1 \log$ $\mathrm{CFU} / \mathrm{g}$ in 5 samples. In study by Silverio et al. (2010), eleven samples were contaminated by molds, with average fungal content of $3.7 \times 10^{3} \mathrm{CFU} / \mathrm{g}$. No contamination was observed in salted shell-free peanuts. Despite the lack of official microbiological criteria on peanuts, there is evidence of fungal contamination during peanut production/shipping procedures that may not be eliminated by roasting [14].

A total of 32 samples purchased from snack shops were found to be clean in terms of Salmonella, E-coli and Listeria spp. Ready-to-eat foods contaminated with Salmonella spp. are considered to be harmful to health and/or unsuitable for human consumption and they contravene the food safety requirements [15]. Researchers evaluated microbiologically to detect the levels of total aerobic mesophilic bacteria, coliforms, Staphylococcus aureus and Salmonella spp. and yeasts and moulds in several types of pistachio and pistachio containing products. They reported that unsatisfactory limits of aerobic mesophilic bacteria, coliforms and yeasts and moulds were obtained in $55 \%(15 / 27), 18.5 \%(5 / 27)$ and $22 \%(6 / 27)$ of the samples respectively. Besides, no Staphylococcus aureus were detected [15].

The use of E. coli as a faecal indicator organism is based on the concept that its detection in food or water samples indirectly provides evidence that the sample has been contaminated with faecal material and that pathogenic organisms may also be present [7].

Researchers found that in only one of 148 samples of peanut taken from snack shops in Brazil, $E$. coli was at an undesirable level $(\geq 100 / \mathrm{g})[4]$.

In this study, shell-free roasted peanuts samples purchased from the snack shops was found clean in terms of the pathogens. When salt and heat treatment applied over $4 \%$, existing microorganisms can be damaged. Besides, the water activity value of the product can be reduced to the level that microorganisms can not develop. For this reason, the environment in which they are stored in processed peanuts is safer as long as the relative humidity does not increase and they are not exposed to cross-contamination. 
Evaluation in terms of mold and mycotoxin in peanuts consumed as a snack is less common than in raw peanuts. The evaluation of mold and aflatoxin in snack peanuts was first done in 1987 by Var [16] and the samples were found clean in terms of aflatoxin. Var and Evliya [17] reported that as a result of molding, aflatoxin formation is inevitable, however molds and aflatoxins are mostly damaged as a consequence of thermal processing with dry salt. Hence, the small amount of aflatoxin left in the samples after thermal treatment could not be in detection limits, consequently the spots were difficult to identify.

Shelled peanut samples collected from harvest and storage were found to be contaminated in terms of microorganisms, however microbiological contamination of shell free same samples were found to be less. In addition, in terms of microorganism it was observed that the shell free samples taken from storage were dirtier than the samples which was being removed the shell taken from storage. According to this, peanut shells are natural protective structures and internal seeds can be preserved as long as mechanical and insect damages do not occur. However, the excess of microbial load on the shells can be transmitted to the product if it is not observed after shell removal and processing. For this reason, the hygienic quality of equipment and the processing environment is becoming important. Besides, the storage conditions are important in terms of the internally stored peanuts, and the appropriate temperature, relative humidity and hygienic conditions must be observed. Peanuts can be stored under conditions that maintain moisture below $10 \%$. Temperature should be maintained between 0 and $10^{\circ} \mathrm{C}\left(32-50{ }^{\circ} \mathrm{F}\right)$.

\section{Conclusions}

Although this is the first large-scale research study on the microbiological assessment of peanuts in Turkey, there is a study where the microbiological quality of other nuts including the roasted peanuts was done. Var et al. [18] were studied a total of 50 nut samples including walnut kernel, peanut, hazelnut, almond, and shelled pistachios. Of the 50 samples, 40 were found to be contaminated with the microorganisms investigated except Salmonella spp. and yeast. Both coliform bacteria and E.coli were found at the range of 3.6 to $1100 \mathrm{MPN} / \mathrm{g}$. Staphylococcus aureus was found as $5 \times 10^{1}-2 \times 10^{2} \mathrm{CFU} / \mathrm{g}$. Molds and total aerobic bacterial count were at the range of $5 \times 10^{1}-1 \times 10^{2} \mathrm{CFU} / \mathrm{g}$ and $5 \times 10^{1}-36 \times 10^{2} \mathrm{CFU} / \mathrm{g}$, respectively. The presence of suspicious Listeria colonies were seen in 3 of the samples including walnut kernels and peanuts.

These studies are preliminary studies and have been studied by the government to encourage routine inspections of these products and to start auditing in state laboratories. Although there was no found Salmonella spp. causing microbiological outbreaks in peanuts in this study, presence of E.coli and Listeria spp. in shelled peanuts is important. The absence of such a consumption in another country does not create problems but in our country the crusts of nuts in the mouth are eaten by breaking. Therefore, this situation makes routine evaluation of this product in our country important.

Control measures to prevent bacterial cross-contamination of raw and processed nuts require procedures for maintaining the hygienic quality of the processing environment and equipment [6]. Application of good agricultural, manufacturing and storage practices together with the hazard analysis and critical control points (HACCP) system that encompass all stages of production, processing and distribution will help ensure the microbial safety of edible nut kernels.

\section{Conflict of Interest}

The authors declare that there is no conflict of interest.

\section{Acknowledgements}

The authors would like to thank General Directorate of Agricultural Research and Policies which funded this project, and Department of Food Engineering at the University of Cukurova which provided of laboratory equipments and all kinds of facilities. 


\section{References}

[1] H.W. Huang, B.B. Yang, C.Y. Wang, Effects of high pressure processing on immunoreactivity and microbiological safety of crushed peanuts, Food Control. 42 (2014) 290-295.

[2] T. Blessington, E.J. Mitcham, L.J. Harris, Survival of Salmonella enterica, Escherichia coli O157:H7, and Listeria monocytogenes on inoculated walnut kernels during storage, Journal of Food Protection, 75(2) (2012) 245-254.

[3] P.K. Brar et al., Survival of Salmonella, Escherichia coli O157:H7, and Listeria monocytogenes on raw peanut and pecan kernels stored at $-24,4$, and $22^{\circ} \mathrm{C}$, Journal of Food Protection. 78(2) (2015) 323-332.

[4] W.J. Song, D.H. Kang, Influence of water activity on inactivation of Escherichia coli O157:H7, Salmonella Typhimurium and Listeria monocytogenes in peanut butter by microwave heating, Food Microbiology. 60 (2016) 104-111.

[5] A.S. Chang, A. Sreedharan, K.R. Schneider, Peanut and peanut products: A food safety perspective, Food Control. 32 (2013) 296-303.

[6] Codex Alimentarius Commission (CAC), Recommended international code of hygienic practice for tree nuts. CAC/RCP 6-1972, (1972). Available: www.codexalimentarius.net/download/standards/266/CXP_006e.pdf.

[7] C.L. Little et al., Survey of Salmonella contamination of edible nut kernels on retail sale in the UK, Food Microbiology. 27 (2010) 171-174.

[8] Anonymous, Merck Gida Mikrobiyolojisi, Ankara, Turkey, 2007.

[9] K. Halkman, H. Doğan, M. Rahati Noveir, Gıda maddelerinde Salmonella ve E.coli aranma ve sayılma yöntemlerinin karşılaştırılması. Gıda Teknolojisi Derneği Yayın no; 21. Ankara. (1994).

[10] Anonymous, Merck Gida Mikrobiyolojisi, Ankara, Turkey, 1998.

[11] I. Var et al., Adana'da Satışa Sunulan Tavuk Etlerinde Listeria spp. Varlığının Araştırılması. 7.Gıda Mühendisliği Kongresi, Konya. s:46 (2012).

[12] FDA, Detection and Enumeration of Listeria monocytogenes in Foods. Bacteriological Analytical Manual, 8th Edition, Revision A. (Erişim tarihi: 12.07.2014). (1998).

[13] S.J. Kenney, L.R. Beuchat, Survival, growth, and thermal resistance of Listeria monocytogenes in products containing peanut and chocolate, Journal of Food Protection. 67(10) (2004) 2205-2211.

[14] A. Silverio, M.M. Lopes, G. de Freitas, Determination of fungal contamination, aflatoxin B1 and ochratoxin A content in peanuts available in Portuguese market, Journal of Biotechnology. 150S (2010) S1-S576.

[15] M. Al-Moghazy, S. Boveri, A. Pulvirenti, Microbiological safety in pistachios and pistachio containing products, Food Control. 36 (2014) 88-93.

[16] I. Var, Çerezlik yerfıstıklarında aflatoksin saptanması üzerine bir araştırma, Çukurova Üniversitesi, Fen Bilimleri Enstitüsü YL tezi. Adana, 1987.

[17] I. Var, B. Evliya, Çerezlik yerfıstıklarında aflatoksin saptanması üzerine bir araştırma, Gıda dergisi. 13(3) (1988) 217-222.

[18] I. Var et al., The microbiological quality of edible nuts, International Conference on Raw Materials to Processed Foods, Antalya, s:53, 2018. 